\title{
A MICROFLUIDIC DEVICE COMBINING DIELECTROPHORESIS AND FIELD FLOW FRACTIONATION FOR PARTICLE AND CELL DISCRIMINATION
}

\author{
Peter Gascoyne, Xiaobo Wang and Jody Vykoukal \\ Section of Experimental Pathology, University of Texas M.D. Anderson Cancer Center \\ Houston, Texas 77030 \\ Harold Ackler, Stefan Swierkowski and Peter Krulevitch \\ Electronics Engineering Technology Division, Lawrence Livermore National Laboratory \\ Livermore, CA
}

\begin{abstract}
The technique of dielectrophoretic field flow fractionation (DEP/FFF) is described and a microfluidic device incorporating this principle is demonstrated. In operation, the device positions particles within a pressure-driven hydrodynamic flow profile by dielectrophoretic levitation according to their dielectric properties. Particles having different dielectric properties are differentially levitated and transported at different rates thereby separating them.
\end{abstract}

\section{INTRODUCTION}

The discrimination and separation of cells and other particulate matter is a central requirement if microfluidic devices are to reach their full potential in biomedical applications. One effect that can be readily exploited for particle manipulation and discrimination on the microscale is dielectrophoresis (DEP). This phenomenon arises when an applied electrical field induces electrical polarization in particles and spatial inhomogeneities in the field distribution act on this induced polarization to produce translational electrostatic forces. DEP is an appealing force for microscale applications because it depends only on the dielectric properties of the target particles and of their suspending medium. The required inhomogeneous electrical fields can be produced by easily fabricated electrodes and the electrical signals needed are of the order of only one volt. Furthermore, unlike electrophoresis, DEP utilizes high frequency $\mathrm{AC}$ electrical fields and electrolytic products are not a concern. Finally, through appropriate choice of the frequency of the applied field, frequency-dependent differences in the dielectric properties of particle subpopulations can be exploited for their sorting and separation from mixtures.

Here, a separation device is presented that utilizes hyperlayer field flow fractionation (FFF) in which particles are separated by being maintained by an applied force field at differential positions within a pressure-driven parabolic hydrodynamic flow profile. In our case, DEP and gravitational forces are balanced so as to accomplish this positioning. Different particle types emerge from the device at times that reflect their respective positions within the flow profile in accordance with their dielectric properties.

\section{THEORY}

A particle in a non-uniform, stationary AC electric field will experience a dielectrophoretic force

$$
\langle F(t)\rangle=2 \pi \varepsilon_{m} r^{3} \operatorname{Re}\left(f_{C M}\right) \nabla E^{2}(r m s)
$$

where the function $\nabla E^{2}(r m s)$ describes the spatial inhomogeneity of the applied AC electrical field. The factor $f_{C M}$ is the well-known Clausius-Mossotti factor

$$
f_{C M}\left(\varepsilon_{p}^{*}, \varepsilon_{m}^{*}\right)=\frac{\varepsilon_{p}^{*}-\varepsilon_{m}^{*}}{\varepsilon_{p}^{*}+2 \varepsilon_{m}^{*}}
$$

which describes the polarizability of the particle in terms of its complex dielectric permittivity $\varepsilon_{p}^{*}$ and that of its suspending medium, $\varepsilon_{m}^{*}$. If the particle has a polarizability less than that of the suspending medium, the DEP force is negative, meaning that the particle is repelled from high field regions. Under these conditions and utilizing an electric field produced by an electrode array on the bottom wall of our device, a DEP levitation force is created on the particle. The particle therefore rises to the position where the DEP levitation force, which is height dependent, balances the gravitational sedimentation force. The equilibrium height is

$$
h_{e q}=\frac{d}{2 \pi} \ln \left\{\frac{3 \varepsilon_{m} U^{2} A p}{2\left(\rho_{c}-\rho_{m}\right) g} \operatorname{Re}\left(f_{C M}\right)\right\}
$$

for a parallel electrode geometry, where $U$ is the electrical potential applied to the electrode array, $\boldsymbol{A}$ is a geometrical term, $\boldsymbol{p}$ is the proportion of the applied field that is unscreened by electrode polarization, and $\left(\rho_{c}-\rho_{m}\right) g$ is the sedimentation force.

If the particle suspending fluid is flowing, a hydrodynamic flow profile will be present. Unless steps are taken to influence the profile, it will be parabolic in form and the fluid velocity at the height $\boldsymbol{h}_{e q}$ is given by

$$
v_{p}=6\langle v\rangle \frac{h_{e q}}{H}\left(1-\frac{h_{e q}}{H}\right),
$$

where $\mathrm{H}$ is the thickness of the chamber and $\langle v\rangle$ is the mean fluid velocity. Particles having different dielectric properties are levitated to different heights and therefore carried along at different rates by the flow profile. In this way, a "mixture of particles becomes separated as it is carried through the chamber. This DEP/FFF principle is illustrated in Figure 1.

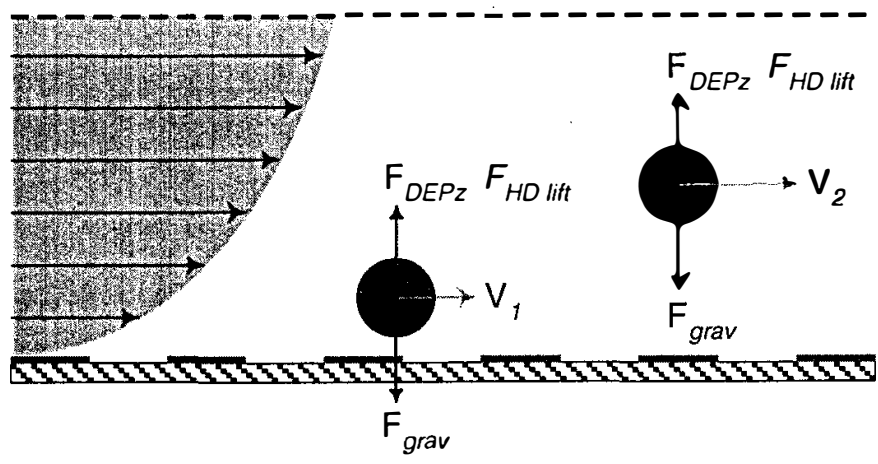

Figure 1. Forces on particles during DEP/FFF separations. Particles having different dielectric properties are levitated by the DEP electrode to different equilibrium heights within the hydrodynamic flow profile (represented by the vectors on the blue area) and are carried through the chamber at different rates. 


\section{TYPICAL RESULTS}

Using polystyrene beads as test particles, the properties of DEP/FFF separators made with microelectrode arrays were characterized and the expressions given in the theoretical section were verified for frequencies of the applied electrical field at which electrode polarization effects could be ignored. Figure 2 shows the very clean fractionation that was obtained for two types of polystyrene beads having similar sizes and densities but different surface properties (non-functionalized versus carboxylated beads). Similarly clean discrimination was observed for other types of beads. Currently we are investigating beads activated to respond to target agents as well as undertaking cell discrimination experiments in diagnostic applications.

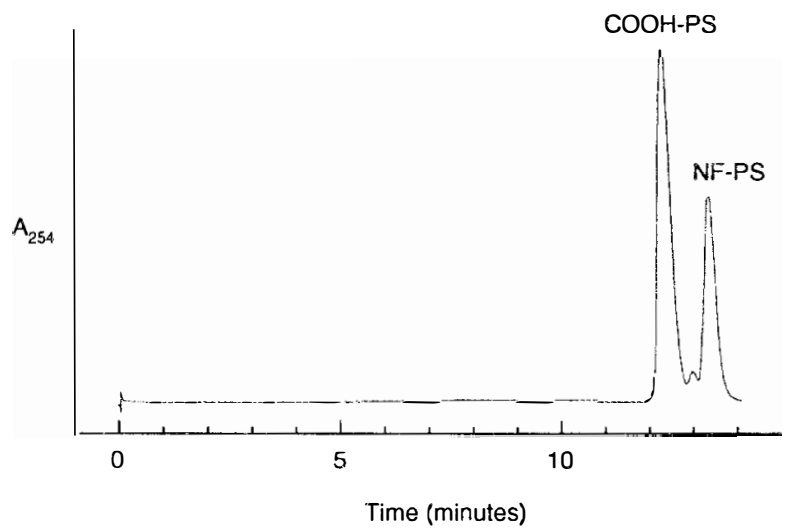

Figure 2. DEP/FFF fractogram illustrating the separation of nonsurface-functionalized polystyrene micro beads from similar micro beads having a carboxylated surface. A mixture of the two bead types was loaded near the inlet of the separation chamber, fluid flow was initiated, and beads leaving the exit port were detected with an ultraviolet sensor (by measuring the absorbance at 254 $\mathrm{nm}$ ). The suspending medium was a sucroseldextrose buffer of conductivity $10 \mathrm{mS} / \mathrm{m}$ and an applied voltage of $1.24 \mathrm{~V}$ RMS at a frequency of $50 \mathrm{kHz}$ was used. While the two types of beads had similar sizes and densities, their different surface characteristics lent them distinguishable dielectric properties at $50 \mathrm{kHz}$.

\section{MICROFLUIDIC IMPLEMENTATION}

The discriminating ability of a DEP/FFF separator depends on the length of the separation chamber. This demands that the chamber be made as long as practical. In the microfabricated implementation presented here, this length criterion is satisfied within a practical amount of real estate by utilizing a serpentine geometry for the separation channel. In this way a $100 \mathrm{~mm}$ channel occupies $3.5 \mathrm{~cm}^{2}$. The serpentine configuration affords a secondary advantage in that the channel can be laid over a simple electrode array so that the channel passes over each electrode element several times. Devices were fabricated by bonding two borosilicate glass (Pyrex) substrates. A 200 micron deep separation channel was etched into one substrate using a wet isotropic etch. One $\mathrm{mm}$ holes were ultrasonically drilled through the outer substrate to provide inlet and outlet ports and electrical contacts. The electrode pattern was then etched from a sputter-deposited chrome/gold layer on this substrate using a mask of electrodeposited resist. Finally, the two substrates were bonded together to form the DEP/FFF separation chamber using a vacuum hot press at $267 \mathrm{psi}$ and $600^{\circ} \mathrm{C}$. A photograph of a two-separator version of such a device is shown in Figure 3.

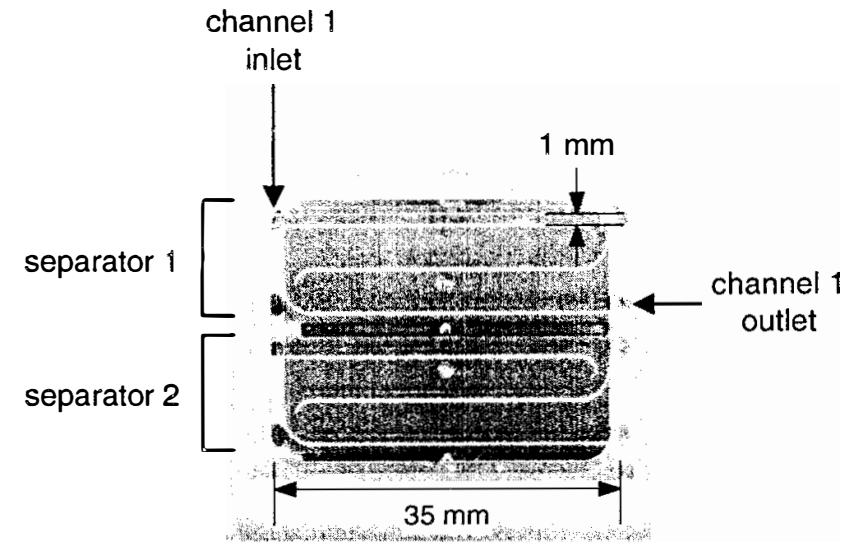

Figure 3. Microfluidic implementation of a dual DEP/FFF separator device. Two separators are fabricated next to one another. Each of the separators has three straight segments and two turns. The device is shown from below within its test package. On separator 1, PEEK tubing (red) that brings fluids in and out of the ports on the top side is visible through the bonded glass body of the device. The darker area covering the separators is an electrode array consisting of two groups of 350 parallel gold elements 50 microns in width and having 50 micron spacing. These electrodes provide the dielectrophoretic levitation force by which particles are discriminated in the flow stream.

\section{CONCLUSIONS}

The technique of dielectrophoresis combined with field flow fractionation has been described. The method is able to discriminate between beads having different surface functionalities and is being tested in detection and cellular diagnostic applications. The glass bonding method affords a straightforward approach to the fabrication of microfluidic devices utilizing this technique. DEP/FFF is an attractive microscale technology because it requires no moving parts except the fluids themselves, it uses AC fields that eliminate problems associated with electrolysis, and it does not require particles to touch any surfaces of the separation chamber.

\section{REFERENCES}

Gascoyne PRC, Y Huang, X Wang, J Yang, G De Gasperis and XB Wang 1996 Cell separation by conventional dielectrophoresis combined with field-flow-fractionation Biophys. J. 70: A.333.

Huang Y, X-B Wang, F F Becker and PRC Gascoyne 1997 Introducing dielectrophoresis as a new force field for field-flow. fractionation Biophys. J. 73: 1118-1129.

Markx GH, J Rousselet and R Pethig 1997 DEP-FFF: field-flowfrcationation using non-uniform electrical fields J. Liq. Chrom \& Rel. Technol. 20: 2857-2872.

Wang X-B, MP Hughes, Y Huang, F F Becker and PRC Gascoyne 1995 Non-uniform spatial distributions of both the magnitude and phase of AC electric fields determine dielectrophoretic forces Biochim. Biophys. Acta 1243: 185-194.

\section{ACKNOWLEDGEMENTS}

This work was supported by the Defense Advanced Research Projects Agency under Contract N66001-97-C-8608 from the Space and Naval Warfare Systems Command. The microfabrication work was conducted under the auspices of the U.S. Dept. of Energy by Lawrence Livermore National Laboratory, contract number W-7405-ENG-48. 